\title{
Early stage small cell lung cancer: very rare, but a surgical disease!
}

\author{
Eric Vallières
}

Division of Thoracic Surgery, Swedish Cancer Institute, Seattle, WA, USA

Correspondence to: Eric Vallières, MD, FRCSC. Division of Thoracic Surgery, Swedish Cancer Institute, 1101 Madisson, Suite 900, Seattle, WA 98104, USA. Email: eric.vallieres@swedish.org.

Provenance: This is an invited Editorial commissioned by the Section Editor Dr. Qiuyuan Li (Department of Thoracic Surgery, Tongji University, Shanghai, China).

Comment on: Wakeam E, Acuna SA, Leighl NB, et al. Surgery Versus Chemotherapy and Radiotherapy For Early and Locally Advanced Small Cell Lung Cancer: A Propensity-Matched Analysis of Survival. Lung Cancer 2017;109:78-88.

Submitted Sep 16, 2017. Accepted for publication Sep 20, 2017.

doi: 10.21037/jtd.2017.09.106

View this article at: http://dx.doi.org/10.21037/jtd.2017.09.106

More than $97 \%$ of patients diagnosed with small cell lung cancer (SCLC) present with very advanced loco-regional and often systemic involvement, a group of patients in whom surgery has no role to play save the odd case where the surgeon may be asked to provide additional tissue to confirm the diagnosis. Less than $3 \%$ of SCLC patients however will be diagnosed at an early stage (N0-N1) and the role of surgery in these unusual SCLC patients remains underutilized.

Over the years, many small series, mostly retrospective, have shown excellent survival for stage I SCLC patients who were treated surgically. Though in many cases these individuals had undergone resection without a diagnosis of SCLC histology prior to surgery, the fact has repeatedly remained that this very small sub-population of SCLC patients do very well after good surgery combined with, since the late 1970s, adjuvant cisplatin based chemotherapy. In many centers however, chemoradiation therapy alone remains the only option offered.

In the absence of randomized studies evaluating the best local modality to treat these rare early stage tumors, case series and small phase II studies have historically guided us in their management. Wakeam et al. now provide us with a propensity-matched analysis of survival where the strategy of surgery and adjuvant chemotherapy was compared to that of chemoradiation therapy for the treatment of early and locally advanced SCLC (1). The surgical cohort identified represented $1.3 \%$ of all SCLC registered in the US National Cancer Data Base in between 2004 and 2013: 2,089 surgical patients, 1,310 with clinical stage I, 335 clinical stage II and 401 with clinical stage III.

This analysis confirmed nearly a double in median overall survival for N0 patients treated with surgery: $38 \mathrm{vs}$. 22 months. Their analysis also showed that the benefits of surgery were only seen when an R0 resection was achieved, that lobectomy was superior to sublobar resections and to pneumonectomies and that adjuvant chemotherapy should be considered after these $\mathrm{R} 0$ resections. These findings had all been hinted previously in smaller published series or non-randomized prospective trials, the size of the cohorts reported here re-enforces these principles.

From my point of view, in 2017, despite the absence of phase III randomized trials, it is more than reasonable to offer primary resection to these patients as strongly suggested by the results of this analysis. These patients however should all get invasive mediastinal staging and brain imaging upfront. We have reported previously that there is a significant discrepancy in between clinical and pathological staging in these patients with $20 \%$ of clinical stage I-II patients being upstaged as well as up to one third of clinical stage III patients being downstaged after resection (2).

As suggested by the observations of Wakeam et al., the extent of resection should probably be at least a lobectomy, though in patients with limited pulmonary reserves for which tumor an anatomical segmentectomy is feasible, without oncological compromise, such an anatomical limited resection may possibly be a good option. The role of surgery when a pneumonectomy is required is probably very limited.

Whatever the size of the lesion, even with pN0 disease, 
adjuvant chemotherapy should be considered standard of care for all resected SCLC patients. The role of postoperative radiation therapy is less well defined and our approach has been to offer it sequentially to chemotherapy in most patients with resected $\mathrm{pN}+$ disease, any $\mathrm{pN}+$ involvement, even when an R0 resection was achieved.

SCLC is usually associated with a very rapid growth rate and early dissemination to regional lymph nodes and distant sites. Are these early SCLCs a biologically distinct population despite apparent similar histology? There may be some merit in pooling our limited banked resected SCLC to evaluate their genetic make-up and evaluate whether genomics could help us better identify those in whom surgery should be considered in the future.

The surgical cohort studied by Wakeam et al. was collected in between 2004 and 2013. It is humbling to note that the survivals we reported after surgery for SCLC collected one decade earlier by the International Association for the Study of Lung Cancer (IASLC) Lung Cancer Staging Project had very similar survivals of around 55\% for resected pathological stages I SCLC, $40 \%$ for pathological stage II and $12 \%$ for pathological stage III.

Cite this article as: Vallières E. Early stage small cell lung cancer: very rare, but a surgical disease! J Thorac Dis 2017;9(11):4185-4186. doi: 10.21037/jtd.2017.09.106

\section{Acknowledgements}

None.

\section{Footnote}

Conflicts of Interest: The author has no conflicts of interest to declare.

\section{References}

1. Wakeam E, Acuna SA, Leighl NB, et al. Surgery Versus Chemotherapy and Radiotherapy For Early and Locally Advanced Small Cell Lung Cancer: A Propensity-Matched Analysis of Survival. Lung Cancer 2017;109:78-88.

2. Vallières E, Shepherd FA, Crowley J, et al. The IASLC Lung Cancer Staging Project: proposals regarding the relevance of TNM in the pathologic staging of small cell lung cancer in the forthcoming (seventh) edition of the TNM classification for lung cancer. J Thorac Oncol 2009;4:1049-59. 